Post-Print author accepted manuscript

\title{
Are missed appointments in an outpatient clinic for adults with congenital heart disease the harbinger for care gaps?
}

Goossens E., Van Deyk K., Budts W.,Moons P.

N.B.: When citing this work, please cite the original article

Reference to published manuscript:

Goossens E., Van Deyk K., Budts W., Moons P. (2021) Are missed appointments in an outpatient clinic for adults with congenital heart disease the harbinger for care gaps? European Journal of Cardiovascular Nursing; doi: 10.1093/eurjcn/zvab012 


\section{Abstract}

\section{Background}

Life-long follow-up is vital for patients with congenital heart disease (CHD) to safeguard longevity. Missing scheduled appointments might be prodromal to subsequent care gaps, but our understanding of their occurrence and impact is limited.

\section{Aims}

This study determined the occurrence and predictors of missed appointments (MA), assess its predictive value for care gaps and explored if MA or care gaps are associated with increased mortality.

\section{Methods}

From 2007-2009, the occurrence of MA in 2,075 CHD patients was documented at an adult CHD clinic. The frequency of outpatient visits in 1,363 adults with moderate to complex CHD was calculated from 2009-2012. Patients without a visit were considered to have a care gap. Data on mortality were collected from 2013 to 2017.

\section{Results}

Missed appointments occurred in $11 \%$ of patients and were more common in men $(\mathrm{OR}=1.57 ; 95 \% \mathrm{Cl}$ : 1.18-2.08), without a history of cardiac procedures (OR=1.46; $95 \% \mathrm{Cl}: 1.08-1.97)$, and for morning visits (OR=1.45; 95\%Cl: $1.10-1.92)$. Care gaps were identified in $6 \%$ of moderate to complex CHD. A MA was significantly associated with an increased likelihood of care gaps $\left(\mathrm{OR}=19.55 ; 95 \% \mathrm{Cl}: 11.92-32.07, \mathrm{R}^{2}=\right.$ 26.5\%). In moderate to complex CHD, no difference in mortality rates was related to the occurrence of discontinued care.

\section{Conclusion}

The occurrence of MA was related to patients' gender, no history of cardiac interventions, and time of day of the outpatient visits. Care gaps were associated by the preceding MA. No association with increased mortality rates was observed. Interventions reducing missed appointments, such as SMSreminders prior to visits, need investigation. 


\section{Introduction}

Despite medical or surgical treatments during childhood, the majority of patients born with congenital heart disease (CHD) cannot be considered as fully cured. Adherence to lifelong follow-up is crucial to avoid long-term complications and safeguard longevity in these patients (1-4). International guidelines prescribe how often patients with CHD should have cardiac follow-up visits at different levels of care $(2,4-7)$. For patients with a simple heart defect, follow-up visits at non-specialist level every 3 to 5 years are deemed sufficient. Patients affected by moderately complex heart defects should present for a follow-up visit every 12 to 24 months, whereas patients with a heart lesion of great complexity ought to receive specialized follow-up care every 6 to 12 months $(4,7)$. These cardiac follow-up visits aim to anticipate early, even pre-symptomatic, deterioration of the clinical status, to manage comorbidities and complications, and to provide patients with state-of-the-art care.

Irrespective the need for continuous follow-up, prior studies found that 7-76\% of patients with CHD had some type of discontinuity of cardiac surveillance $(8,9)$. The consequences of such discontinuity of care are far-reaching $(3,10,11)$. One study, conducted in the USA, reported that $60 \%$ of patients ( $n=99$ ) who have had lapses of care presented with a new diagnosis of hemodynamic significance, including significant valvular regurgitation $(41.0 \%, n=39)$, obstructive lesions $(21.0 \%$, $\mathrm{n}=20)$, ventricular dysfunction $(18.0 \%, \mathrm{n}=17)$, new anatomical lesions $(11.0 \%, \mathrm{n}=10)$, pulmonary hypertension $(5.0 \%, n=5)$, and coronary obstructive lesions $(4.0 \%, n=3)(3)$. Furthermore, these patients had a 3.1 times greater likelihood of needing an urgent surgical or catheter-based intervention (3). A Danish study found that $56 \%$ of 18 patients with tetralogy of Fallot considered to be lost to follow-up had moderate to severe pulmonary valvular regurgitation; $75 \%$ of 8 patients with an atrioventricular septal defect had a moderate to severe regurgitation of the common atrioventricular valve; and one in five patients with coarctation of the aorta presented with a significant re-coarctation (10). A third study, performed in the Netherlands, reported that $16 \%$ of 593 patients who have been lost to follow-up, presented with newly diagnosed complications during their cardiac examination of whom $6 \%$ needed prompt interventions (12).

One of the strongest predictors for discontinuity of care in patients with CHD appeared to be a history of one or more missed appointments. Missed appointments (MA) are defined as a "no-show of the patient for a scheduled outpatient visit without sufficient notification, or any notification at all" (13) and occur in $15.5 \%$ to $26.0 \%$ of planned visits in CHD patients $(11,14)$. Patients who had at least one MA in the past had a 13 -fold likelihood (95\% Cl: 3.30-51.70) of experiencing a care gap with a duration of at least three years (13). This odds ratio is substantially higher than that of other patient- 
related predictors for care gaps (15). Hence, MA are not only a common problem generating inefficiencies in outpatient clinics and yielding a waste of professionals' time, it also may be a strong harbinger for future care gaps. The evidence base of the predictors and consequences of MA, however, is limited to studies performed predominantly in children, adolescents and other patient populations (16-19). The objectives of the present study were therefore (i) to determine the proportion of missed appointments (MA) at a large-volume adult CHD outpatient clinic; (ii) to identify predictors of MA; (iii) to explore if MA predict future care gaps; and (iv) to explore if MA or care gaps are associated with increased mortality rates in adults with moderate to complex $\mathrm{CHD}$.

\section{Methods}

\section{Study design}

We conducted a descriptive, observational study. A schematic representation of the study design is provided in Figure 1. The study protocol conforms to the ethical guidelines of the 1975 Declaration of Helsinki as reflected in a priori approval by the local ethics committee of the University Hospitals Leuven (20). Informed consent was neither required nor sought because a clinical team member (KVD) retrospectively extracted data.

\section{PLEASE INSERT FIGURE 1 ABOUT HERE}

\section{Setting}

The study was performed at the adult CHD outpatient clinic of the University Hospitals Leuven, Belgium. This adult CHD program oversees the follow-up of patients with congenital and structural heart defects as of the age of 16 years. Nowadays, more than 3,500 outpatient visits are recorded annually. Over the past decade, the ACHD clinic was growing at an average rate of 100-150 outpatient visits per year.

At the adult CHD clinic, it is standard practice that patients with CHD receive a written invitation letter for every scheduled appointment with an adult CHD cardiologist, according to the follow-up frequency that was agreed upon (9). Patients, who failed to show up for their scheduled appointment, receive an invitation letter for a new appointment as soon as possible. This pro-active strategy is repeated up to a maximum of three times after patients' initial MA.

\section{Study population}

Inclusion criteria were: diagnosed with CHD as defined by Mitchell et al. (21); having had a scheduled outpatient visit at the adult CHD outpatient clinic between June 2007 and June 2009; and 
living in Belgium during the study period. Exclusion criteria were: not having had a heart transplantation and/or not being deceased at the moment of the initial scheduled outpatient visit.

\section{Outcome measures}

In line with a previous study, MA was defined as a "no-show of the patient for a scheduled outpatient visit without sufficient notification, or any notification at all (13)". Patients who notified the hospital about their inability to show up for their upcoming visit, even if it was only a few hours prior to the appointment, were not considered to have an MA.

Furthermore, care gaps were conceptually defined as "any discontinuation of the care process where the time lapse between mandatory visits exceeded the recommended period of time" (15). Ascertaining care gaps was done in patients with moderate or highly complex heart defects (1) and who were still alive in the observation period of July 2009 - December 2012. The reason to include only patients with moderate to complex defects is that discontinuity of care places these patients at greater risk for missed changes in health, compared to mild heart defects. Given that guidelines suggest that patients with moderately complex heart defects should receive cardiac follow-up at least every 3 years $(4,7)$ a recommendation that is followed in our center, an observation period of 3.5 years to assess care gaps is deemed appropriate.

\section{Data collection}

For every clinic day, a list of patients with scheduled appointments was made. During a two-year period (June 2007-June 2009), notes were collected on these lists indicating whether or not patients showed up for their scheduled visit. Based on these notes, the occurrence and number of missed appointments was determined.

Data on the occurrence of care gaps were collected based on the Hospital Information System between July 2009 and December 2012 (i.e., time interval of 3.5 years). Patients were identified with a care gap if no outpatient visit at this specific ACHD clinic was registered in the administration.

Additional demographic (e.g., age and sex) and clinical variables (e.g., type of heart defect and history of catheter or surgical interventions) were retrospectively collected from the patients' medical records. The anatomical complexity of the heart defect was categorized into three groups (i.e., simple, moderate, and complex heart disease) according to the criteria of Task Force 1 of the $32^{\text {nd }}$ Bethesda Conference $(1,7)$ 
From January 2013 until July 2017, based on chart review data were collected on the date and cause of death in patients with moderate to complex CHD. In case of censoring of patients, the date at which the patient had the last contact with healthcare providers within the hospital was collected from the medical record.

\section{Statistical analysis}

Data were analyzed using IBM ${ }^{\circledR}$ SPSS $^{\circledR}$ Statistics 24.0 (Armonk, NY: IBM Corp). Nominal variables were expressed in terms of absolute numbers and percentages. For continuous variables, medians and quartiles are calculated due to the observation that data were not normally distributed. For inferential statistics, multivariable logistic regression analysis was used to identify demographic, clinical, and organizational characteristics associated with the occurrence of MA (enter method). To investigate if MA predict care gaps, two-step hierarchical multivariable logistic regression analysis was employed. The first step model in this hierarchical analysis, contained a set of 6 demographical, clinical and organizational factors. In order to investigate the amount of variability of care gaps explained by the occurrence of MA in the past, this latter variable was added in the second step model. Nagelkerke's $\mathrm{R}^{2}$ was determined to quantify the amount of explained variability of care gap occurrence by the set of significant predictors. To explore if MA or care gaps are associated with increased mortality rates in adults with moderate to complex CHD, a Fisher's Exact Test was performed.

\section{Results}

\section{Sample characteristics}

In the two-year data collection period for MA, 3,432 outpatient visits were scheduled for a total of 2,075 unique patients. These patients had a median age of 26.0y $\left(Q_{1}=20.0 y ; Q_{3}=35.0 y, \min =15.0 y\right.$; $\max =86.0 \mathrm{y})$ at the time of the first scheduled appointment, and 1,094 (52.7\%) were men. Overall, 683 (32.9\%) patients had a mild heart defect; $1,132(54.6 \%)$ had a moderately complex defect; and 260 $(12.5 \%)$ had a highly complex heart lesion. A history of cardiac surgery was present in 1,252 patients $(60.3 \%)$ and $461(22.2 \%)$ patients underwent a catheter-based intervention in the past.

\section{Prevalence and characteristics of missed appointments}

Overall, $281(8.2 \%)$ of the 3,432 scheduled visits were characterized by a no-show of the patient without any notification (i.e., MA). These MA occurred in 230 (11.1\%) unique patients. More specifically, 183 patients (79.6\%) missed one appointment; 44 patients (19.1\%) missed two subsequent appointments; and 3 patients (1.3\%) failed to show up for three consecutive scheduled appointments. Missed appointments occurred in about one in ten patients, irrespective of the CHD complexity as 
$11.7 \%$ of 683 patients with mild CHD, $10.5 \%$ of 1132 patients with moderate CHD and $11.9 \%$ of 260 patients with complex CHD, respectively were found to have at least one MA ( $\chi^{2}=0.835, p=.659$ ). Within the group of patients with mild CHD who had a missed appointment $(n=80)$, the majority $(n=60$; 75\%) had one MA, while 19 patients (23.8\%) had 2 MAs and one patient (1.25\%) had 3 MAs. In mild CHD, patients who missed an appointment, most often experienced only 1 MA ( $n=95 ; 79.8 \%$ ), while 22 patients $(18.5 \%)$ had two MAs and 2 patients (1.7\%) had three MAs. Within the group of complex CHD, 28 patients (90.3\%) had one MA, 3 patients $(9.7 \%)$ had two MAs and none of the patients missed three appointments. The difference was not statistically significant $(F=3.311 ; p=.470)$. The highest proportion of missed appointments occurred in May (36/344 visits; 10.5\%), while the lowest number was found in December (12/256 visits; 4.7\%). About one out of ten missed appointments happened in the morning (119/1,143 missed appointments; 10.4\%).

\section{Predictors of missed appointments}

Multivariable logistic regression analysis showed that MAs were more common in men than women $(\mathrm{OR}=1.57 ; 95 \% \mathrm{Cl}: 1.18-2.09)$; in patients with no history of cardiac surgery in the past (OR=1.46; $95 \% \mathrm{Cl}$ : 1.08-1.97), and visits scheduled in the morning (OR=1.45; 95\% $\mathrm{Cl}$ : 1.10-1.92) (see Table 1). This model explained only $2.1 \%$ of the variance of MA.

\section{PLEASE INSERT TABLE 1 ABOUT HERE}

\section{Occurrence and predictors of care gaps}

Of the 1,392 patients with either moderate or complex heart lesions, 29 patients (2.1\%) died during the observation period 2009-2012. This means that 1,363 unique patients with a moderate to complex heart lesion could be assessed for care gaps during the subsequent observation period of 3.5 years (2009-2012). A total of 84 patients (6.2\%) was found to have a care gap, of whom 50 patients (57.5\%) had at least one MA in the past. On the other hand, within the group of patients with a care gap, 34 patients $(40.5 \%)$ did not miss an appointment during the preceding period. The characteristics of this group are presented in Table 2 .

\section{PLEASE INSERT TABLE 2 ABOUT HERE}

Eighty-four patients (6.2\%) of the 1,363 patient did not have an outpatient visit in the period 2009-2012, thus were considered to have a care gap of at least 3.5 years. While the first-step model in the hierarchical binary logistic regression analysis only explained $2.1 \%$ of the variance of care gaps, the final second-stage model explained $26.5 \%$. This final model demonstrated that care gaps were more 
prevalent in patients with at least one missed appointment in the period 2007-2009 (OR=19.55; 95\% Cl: 11.92-32.07) (See Table 3). Age, CHD complexity level, history of cardiac surgery or catheter-based interventions and sex, appeared to be non-significant predictors of care gaps.

\section{PLEASE INSERT TABLE 3 ABOUT HERE}

Mortality associated with missed appointments and care gaps in patients with moderate to complex CHD

Over the course of the entire study, i.e., 2009-2017, 75 out of the 2,075 unique patients (3.6\%) died. When focusing on patients with moderate to complex heart defects, 35 out of 1,363 patients (2.6\%) died from January 2013 to July 2017. The difference in mortality between patients with versus without missed appointments was not significant $(4 / 145=2.76 \%$ versus $31 / 1218=2.55 \%$; Fisher's Exact test $\mathrm{p}=.783)$. Furthermore, mortality was observed in only 2 out of 84 patients $(2.4 \%)$ with care gaps in 2009-2012. This proportion was not significantly different to the mortality of patients without care gaps (33/1279=2.6\%; Fisher's Exact Test $p=1.000)$.

\section{Discussion}

Non-attendance at outpatient clinics is a commonly observed problem in patients affected with a congenital and/or chronic condition, occurring in $10 \%$ to $27 \%$ of the scheduled outpatient visits $(11,13,14,22)$. Missed appointments are a major source of inefficiency, as this event disrupts the healthcare delivery system at various points. This phenomenon is worrisome since most patients with CHD do require life-long specialized follow-up care, to avoid long-term complications and safeguard longevity. Hence, we aimed to gain a better understanding of the occurrence, predictors and implications of MA in patients who have scheduled follow-up visits at our ACHD outpatient clinic.

Our study showed that MA occurred in $8 \%$ of scheduled visits, and in $11 \%$ of unique patients who had a scheduled outpatient visit. Our proportion of MA is at the lower end of previously found MA rates in $\operatorname{CHD}(11,14)$ and occurred in our sample more frequently in patients without a history of cardiac surgery, in men, and for morning visits. The predictors assessed, however, only explained about $2 \%$ of the variability in MA, indicating that MA might be better predicted by a multifactorial assessment of different elements such as patients' health beliefs and health status, scheduling problems, or insurance status (22)

Care gaps, operationalized as no outpatient visit at the ACHD clinic in a period of 3.5 years, occurred in $6.4 \%$ of our sample of patients with moderate to complex CHD. This proportion is relatively low as compared to previous studies on lapses of care and lost to follow-up conducted in different 
countries. A recently published meta-analysis showed that rates of discontinuity of care ranged between $3.6 \%$ and $62.7 \%$ (23). Significant geographical variability in estimated proportion of discontinuity of follow-up care in CHD patients was found in literature, exemplified by rather high estimated proportions of care gaps in USA (34.0\%; $95 \% \mathrm{Cl}$ : $24.3-45.4 \%$ ) and Canada $(25.7 \%$; $95 \% \mathrm{Cl}$ : 17.0-36.7\%) and lower proportions in European countries (6.5\%; 95\% Cl: 5.3-7.9\%) (23). However, the current findings are in line with the results of a study in our center some years ago reporting that about $7 \%$ of patients had care gaps within a period of $5 y$ after the transfer from pediatrics to adult facilities (15). In that study, which was performed in all types of $\mathrm{CHD}$, we found that male sex and no prior heart surgery were predictors of 'no cardiac follow-up' (15). Together, these two factors explained $11.4 \%$ of the variance of 'no follow-up' (based on Nagelkerke $\mathrm{R}^{2}$, data on file). At our hospital and ACHD clinic, some structural and organizational elements might have helped to keep discontinuation rates rather low. Within our hospital, it is standard practice to transfer patients from pediatric cardiology towards the ACHD clinic around the age of $16 y$, unless their medical condition is unstable. Since both the pediatric and adult cardiology units are located in the same hospital and share on clinical database, the practical and administrative transfer of patients runs smoothly. Furthermore, a rather pro-active approach is used in terms of sending out invitations and reminders for scheduled appointments. Finally, our practice of sending out up to three reminders to patients who subsequently missed their appointment might already positively impact the occurrence of care gaps.

The current study added to this existing knowledge by showing that MA explained $26.5 \%$ of the variance of care gaps. Indeed, the likelihood of having a care gap was 19 times greater in patients who had an MA in the preceding two years. This makes it the strongest predictor for care gaps in our study, but it also confirms the strong predictive value of MA in CHD (13) and in other chronic conditions (15).

Given that MA is such a strong predictor for future care gaps, MA ought to be monitored in clinical practice and measured to prevent MA and/or keeping patients in the system are essential. Exploration of effective interventions which could be implemented in practice and prevent patients from missing out on scheduled appointments is an important step. Based on the results of the Cochrane meta-analysis performed by Gurol-Urganci et al. (2013), moderate quality evidence is provided that mobile text messages improve the rate of attendance at healthcare appointments compared to no reminders (RR 1.14; 95\%Cl:1.03-1.26) or postal reminders alone (RR 1.10; 95\%Cl: 1.02 1.19) (22). We plan to implement the use of SMS reminders which will be sent out to patient one week prior to their scheduled visit and will evaluate the effectiveness of this intervention on the clinical nonattendance rate at our ACHD clinic accordingly. 
Although we identified a limited set of significant determinants, our list was non-exhaustive. A Cochrane systematic review assessed patient- and healthcare system- related factors leading to MA (22). On the one hand, the appointment-keeping behavior of patients was found to be influenced by demographics such as age, sex, ethnicity and socio-economic status. On the other hand, alternative patient-reported factors for missing appointments were: health beliefs, lack or difficulty of transportation, scheduling problems, patients' health status, resistance to consultation, insurance status and frustration with the outpatient clinic organization. Renewal of drug prescriptions, recent hospitalizations, co-morbidities such as arrhythmias or heart failure, or the occupation of patients could potentially impact the likelihood of attending clinic appointments. Finally, a set of healthcare system factors was found to be of influence such as: inadequate communication between healthcare providers and patients, waiting lists, time between referral and appointments, quality of the consultation, administrative problems, and the site of care (22). Based on this body of knowledge, we understand that prevention of MA is a multidimensional process requiring attention to patient- and organizational factors, and of paramount importance to prevent the occurrence of care gaps.

The results of this study can facilitate us to improve the organization of outpatient care at the adult CHD clinic in order to safeguard continuity of lifelong care. Based on these findings, extra attention should be given to the occurrence of MA in patients. As healthcare providers, we have the responsibility to actively reach out to patients not attending a scheduled follow-up appointment. Patients can, for example, receive an additional reminder through postal mail, telephone or text message and should be actively invited back to the outpatient clinic. Getting hold of up-to-date contact details of patients can be challenging in today's society, depending on the availability of centrally registered contact details and the linkage with the hospital administrative system. Hence, the role of primary care providers, such as general practitioners, in the continuity of care for patients with chronic conditions will gain importance in the future. Moreover, it could be interesting to investigate the effect of virtual clinics or weekend appointments on the occurrence of MA in this population as avenues for the future.

Although Kempny et al. (2016) reported to be the first to demonstrate that adherence to appointments at the outpatient adult CHD clinic was a strong and independent predictor for better survival, our study could not confirm these findings (11). This study, however, employed a longer period for retrospective data collection covering almost 18 years of time. Within our sample including all types of CHD, mortality rates did not differ significantly in patients with or without missed appointments, nor in patients with or without care gaps. Important differences in study methodology are, however, to be kept in mind when comparing our data with those reported by Kempny and 
coworkers. Their study was performed in a sample of $>4,400$ patients with a median age of $26.4 y$, with clinical non-attendance rate reported to be around $23 \%$ and 366 patients $(8.2 \%)$ who died during the course of this longitudinal study with a median follow-up time of 9.6 years per patient.

This study employed a long observation window of 5.5 years for the assessment of MA and care gaps in a large and representative sample of adults with all types of CHD. Based on current guidelines, which are implemented in our ACHD clinic, an observation period of 3.5 years was used for the assessment of care gaps, as care gaps were only assessed in patients with moderate to complex CHD. During this time interval, we kept records about the number of patients who missed their scheduled appointment. Follow-up appointments in patients with complex $\mathrm{CHD}$ are scheduled to occur 1 year after the last visit to the maximum. For patients with moderate CHD, follow-up appointments are scheduled within a period of 3 years at the maximum after their latest visit. Hence, although the observation period used for the assessment of care gaps, spanned a total of 3.5 years, we checked if patients showed up for their scheduled appointment or not. Indirectly, we differentiated between patients with a complex or moderate heart lesion. The descriptive results of our study demonstrated that although the concepts of missed appointment and care gaps might appear to be highly related to each other at first glance, they clearly appeared to be distinguishable. Since only $57 \%$ of patients with a care gap were found to have at least one MA in the past, these variables are conceptually different from each other. Some methodological limitations should, however, be kept in mind when interpreting the results of our study. Firstly, our study was a single center study conducted at one large-volume outpatient adult CHD clinic limiting generalizability of the results. Secondly, while data on the occurrence of missed appointments and mortality was collected prospectively, data on predictors and care gaps were, however, collected retrospectively. Furthermore, if patients informed the hospital they were unable to attend the scheduled appointment, these patients were not considered to have a missed appointment. However, we did not evaluate if patients themselves took the initiative to immediately schedule a new appointment or within another CHD clinic. Furthermore, we have to take into account that some patients might have moved their home address without informing the hospital, potentially leading to missing out on hospital correspondence reminding patients about their scheduled appointment(s). Hence, a slight underestimation of the occurrence of missed appointments might be present if these patients eventually did not reschedule a new appointment, dropping out of routine follow-up care. Finally, although our set of selected determinants explained about $26.5 \%$ of the variability in care gaps, other important patient-, hospital- or healthcare system factors could alter the patients' risk for care gaps.

Future studies using population-based samples collecting information on healthcare use of patients with CHD longitudinally are highly needed in order to examine if this association between 
discontinuity of care and detrimental health effects can be confirmed. Such studies are mandatory in order to enlarge our body of knowledge on the importance of continuous life-long cardiac care in adult CHD patients.

\section{Conclusion}

The occurrence of MA at our adult CHD clinic appeared to be in line with rates observed in other populations of complex chronic conditions. Determination of significant predictors help us identify patients prone to MA and furthermore facilitate us to improve the organization of outpatient care in the ACHD outpatient clinic. The impact of missed appointment can, however, not be underestimated as this event significantly increased the likelihood of subsequent care gaps in patients whom are recommended to have regular and frequent cardiac follow-up visits in order to safeguard longevity and quality of life. Our study demonstrated that missed appointment explained about one-fourth of the variance in care gaps in adults with moderate to complex CHD.

\section{Implications for practice}

1. About one in ten patients with a scheduled visit to the outpatient ACHD clinic was found to have missed at least one appointment during the observation time.

2. Missed appointments occurred more frequently in patients without a history of surgery, in men and for morning visits.

3. Prediction of missed appointment might remain a challenge in practice, as a multifactorial approach was found to be of importance since sociodemographic and clinical variables appeared to predict variability to a limited extent.

4. Having a missed appointment can be considered a harbinger for care gaps, as the likelihood of care gaps was 19 times greater in patients who had a missed appointment in the preceding two years.

\section{Acknowledgements}

The authors want to acknowledge Maaike Beckx, Liesbet van Bulck, and Fouke Ombelet for their valuable contribution during the process of data collection.

\section{Funding sources}

None

\section{Disclosures}

None 


\section{References}

1. Warnes CA, Liberthson R, Danielson GK, et al. Task force 1: the changing profile of congenital heart disease in adult life. J Am Coll Cardiol. 2001;37(5):1170-1175.

2. Foster E, Graham TP, Jr., Driscoll DJ, et al. Task force 2: special health care needs of adults with congenital heart disease. J Am Coll Cardiol. 2001;37(5):1176-1183.

3. Yeung E, Kay J, Roosevelt GE, Brandon M, Yetman AT. Lapse of care as a predictor for morbidity in adults with congenital heart disease. Int J Cardiol. 2008;125(1):62-65.

4. Landzberg MJ, Murphy DJ, Jr., Davidson WR, Jr., et al. Task force 4: organization of delivery systems for adults with congenital heart disease. J Am Coll Cardiol. 2001;37(5):1187-1193.

5. Warnes CA, Williams RG, Bashore TM, et al. ACC/AHA 2008 Guidelines for the Management of Adults with Congenital Heart Disease: a report of the American College of Cardiology/American Heart Association Task Force on Practice Guidelines (writing committee to develop guidelines on the management of adults with congenital heart disease). Circulation. 2008;118(23):e714-833.

6. Deanfield J, Thaulow E, Warnes C, et al. Management of grown up congenital heart disease. Eur Heart J. 2003;24(11):1035-1084.

7. Baumgartner $\mathrm{H}$, Budts W, Chessa $\mathrm{M}$, et al. Recommendations for organization of care for adults with congenital heart disease and for training in the subspecialty of 'Grown-up Congenital Heart Disease' in Europe: a position paper of the Working Group on Grown-up Congenital Heart Disease of the European Society of Cardiology. Eur Heart J. 2014;35(11):686-690.

8. Goossens E, Fernandes SM, Landzberg MJ, Moons P. Implementation of the American College of Cardiology/American Heart Association 2008 Guidelines for the Management of Adults With Congenital Heart Disease. Am J Cardiol. 2015;116(3):452-457.

9. Goossens E, Stephani I, Hilderson D, et al. Transfer of adolescents with congenital heart disease from pediatric cardiology to adult health care: an analysis of transfer destinations. J Am Coll Cardiol. 2011;57(23):2368-7234.

10. Iversen K, Vejlstrup NG, Sondergaard L, Nielsen OW. Screening of adults with congenital cardiac disease lost for follow-up. Cardiol Young. 2007;17(6):601-608.

11. Kempny A, Diller GP, Dimopoulos K, et al. Determinants of outpatient clinic attendance amongst adults with congenital heart disease and outcome. Int J Cardiol. 2016;203:245-250.

12. Vis JC, van der Velde ET, Schuuring MJ, et al. Wanted! 8000 heart patients: identification of adult patients with a congenital heart defect lost to follow-up. Int J Cardiol. 2011;149(2):246-247.

13. Mackie AS, Rempel GR, Rankin KN, Nicholas D, Magill-Evans J. Risk factors for loss to follow-up among children and young adults with congenital heart disease. Cardiol Young. 2012;22(3):307-315.

14. Strijbosch AMMZR, Blom NA, Bouma BJ, et al. Transition from paediatric to adult care of adolescent patients with congenital heart disease: a pathway to optimal care. Neth Heart J. 2016;24:682-690.

15. Goossens E, Bovijn L, Gewillig M, Budts W, Moons P. Predictors of Care Gaps in Adolescents With Complex Chronic Condition Transitioning to Adulthood. Pediatrics. 2016;137(4): e20152413.

16. Chariatte V, Berchtold A, Akre C, Michaud PA, Suris JC. Missed appointments in an outpatient clinic for adolescents, an approach to predict the risk of missing. J Adolesc Health. 2008;43(1):38-45.

17. Murdock A, Rodgers C, Lindsay H, Tham TC. Why do patients not keep their appointments? Prospective study in a gastroenterology outpatient clinic. J R Soc Med. 2002;95(6):284-286.

18. Potamitis T, Chell PB, Jones HS, Murray PI. Non-attendance at ophthalmology outpatient clinics. J R Soc Med. 1994;87(10):591-593.

19. Corfield L, Schizas A, Noorani A, Williams A. Non-attendance at the colorectal clinic: a prospective audit. Ann R Coll Surg Engl. 2008;90(5):377-380.

20. World Medical Association. Declaration of Helsinki: ethical principles for medical research involving human subjects. JAMA. 2013;310(20):2191-2194.

21. Mitchell SC, Korones SB, Berendes HW. Congenital heart disease in 56,109 births. Incidence and natural history. Circulation. 1971;43(3):323-32. 
22. Gurol-Urganci I, de Jongh T, Vodopivec-Jamsek V, Atun R, Car J. Mobile phone messaging reminders for attendance at healthcare appointments. Cochrane Database Syst Rev. 2013(12):CD007458.

23. Moons P, Skogby S, Bratt EL, Zühlke L, Marelli AJ, Goossens E. Discontinuity of cardiac followup in young persons with congenital heart disease transitioning to adulthood. J Am Heart Ass. 2021; In Press. 
Table 1: Predictors of missed appointments identified using binary logistic regression analysis $(n=2,075)$

\begin{tabular}{|c|c|c|c|c|}
\hline Variables & ß & $\operatorname{Exp}(ß)$ & $95 \% \mathrm{Cl}$ & p-value \\
\hline Age at time of outpatient visit & -.002 & .998 & $.986-1.009$ & .698 \\
\hline $\begin{array}{l}\text { Anatomical complexity of } \\
\text { congenital heart disease } \\
\text { (Categorical: mild-moderate- } \\
\text { complex) }\end{array}$ & .085 & 1.089 & $.865-1.371$ & .469 \\
\hline $\begin{array}{l}\text { History of cardiac surgery } \\
\text { (Reference: no history of cardiac } \\
\text { surgery) }\end{array}$ & .375 & 1.455 & $1.075-1.970$ & .015 \\
\hline Sex (Reference: female) & .448 & 1.566 & 1.179-2.079 & .002 \\
\hline $\begin{array}{l}\text { History of catheter-based } \\
\text { intervention(s) (Reference: no } \\
\text { history of catheter-based } \\
\text { intervention(s)) }\end{array}$ & .124 & 1.132 & $.803-1.597$ & .479 \\
\hline $\begin{array}{l}\text { Part of the day at which the visit } \\
\text { was scheduled (reference } \\
\text { afternoon) }\end{array}$ & .373 & 1.452 & 1.097-1.922 & .009 \\
\hline Constant & -2.822 & .059 & & .000 \\
\hline
\end{tabular}

Nagelkerke $R^{2}=2.1 \% ; p$-values $<0.05$ are reported in bold. 
Table 2: Characteristics of subgroup of patients with moderate to highly complex congenital heart disease $(n=1,363)$

\begin{tabular}{lrr}
\hline Variables & n(\%) \\
\hline Sex & Male & $734(53.9)$ \\
& Female & $629(46.1)$ \\
History of cardiac surgery & Yes & $1,037(76.1)$ \\
& No & $326(23.9)$ \\
History of catheter-based intervention(s) & Yes & $326(23.9)$ \\
& No & $1,037(76.1)$ \\
CHD complexity based on Task Force 1 & Moderately complex & $1,109(81.4)$ \\
& Highly complex & $254(18.6)$ \\
Age in years (median, $Q_{1}-Q_{3}$, range) & & $26 y, 20 y-35 y,[15 y-86 y]$ \\
Missed appointment between June 2007-2009 & Tho subsequent MA & $23(15.9)$ \\
Number of missed appointments (MA) & Three subsequent MA & $1(0.7)$
\end{tabular}

$\mathrm{MA}=$ missed appointment; $\mathrm{CHD}=$ congenital heart disease 
Table 3: Predictors of care gaps based on hierarchical binary logistic regression modelling $(n=1,363)$

\begin{tabular}{|c|c|c|c|c|c|c|}
\hline \multicolumn{3}{|r|}{ Variables } & B & $\operatorname{Exp}(ß)$ & $95 \% \mathrm{Cl}$ & p-value \\
\hline \multirow{8}{*}{$\begin{array}{l}\frac{-1}{0} \\
\frac{0}{0} \\
\frac{0}{2}\end{array}$} & \multirow{8}{*}{$\begin{array}{l}\frac{N}{0} \\
\frac{0}{0} \\
\frac{0}{2}\end{array}$} & Age at time of outpatient visit & .000 & 1.000 & $.979-1.021$ & .99 \\
\hline & & $\begin{array}{l}\text { Anatomical complexity of congenital heart disease (categorical: mild- } \\
\text { moderate-complex) }\end{array}$ & -.044 & 0.957 & $.6511-1.793$ & .892 \\
\hline & & History of cardiac surgery (reference: no history of cardiac surgery) & 491 & 1.634 & $.963-2.772$ & .068 \\
\hline & & Sex (reference: female) & -.336 & .715 & $.436-1.171$ & 183 \\
\hline & & $\begin{array}{l}\text { History of catheter-based intervention(s) (reference: no history of catheter- } \\
\text { based intervention(s)) }\end{array}$ & 038 & 1.039 & $.588-1.836$ & .896 \\
\hline & & Part of the day at which the visit was scheduled (reference: afternoon) & -.423 & .655 & $.385-1.113$ & .118 \\
\hline & & $\begin{array}{l}\text { At least one missed appointment during observation period from June 2007- } \\
\text { June } 2009\end{array}$ & 2.973 & 19.552 & 11.919-32.074 & $<.001$ \\
\hline & & Constant & -3.330 & .036 & & $<.001$ \\
\hline
\end{tabular}

Nagelkerke $R^{2}$ of Model 1 = 1.3\%; for Model 2= 26.5\%; $p$-values $<0.05$ are reported in bold. 